\title{
A Poly(ethylene glycol)-modified Tapered-Slit Membrane Filter for the Efficient Release of Captured Viable Circulating Tumor Cells
}

\author{
Young Jun $\mathrm{Kim}^{\dagger}$, Yoon-Tae Kang ${ }^{\dagger}$, and Young-Ho Cho*
}

Department of Bio and Brain Engineering, Korea Advanced Institute of Science and Technology (KAIST),

291 Daehak-ro, Yuseong-gu, Daejeon 305-701, Republic of Korea

\section{Contents}

S1. The modification of the tapered-slit filters (TSFs)

S2. XPS Analysis.

S3. Purity Test

S4. Surface Condition Analysis S-5

S5. Cell Capturing Appearance. ..S-6

S6. RT-PCR Analysis S-7 


\section{S1. The modification of the tapered-slit filters (TSFs)}

The entire process of fabrication and modification is described below:

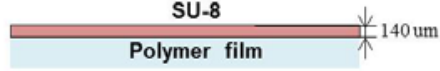

(a) SU8 spin coating on polymer film

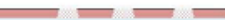

(b) UV exposure

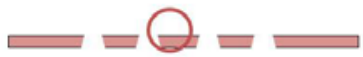

(c) Development and polymer peeling-off

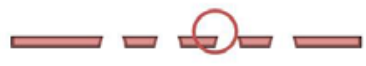

(d) Acid treatment

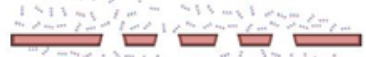

(e) Self-assembly of PEG-silane molecules

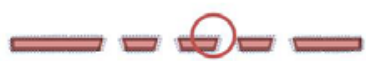

(f) Washing and removing excess molecules
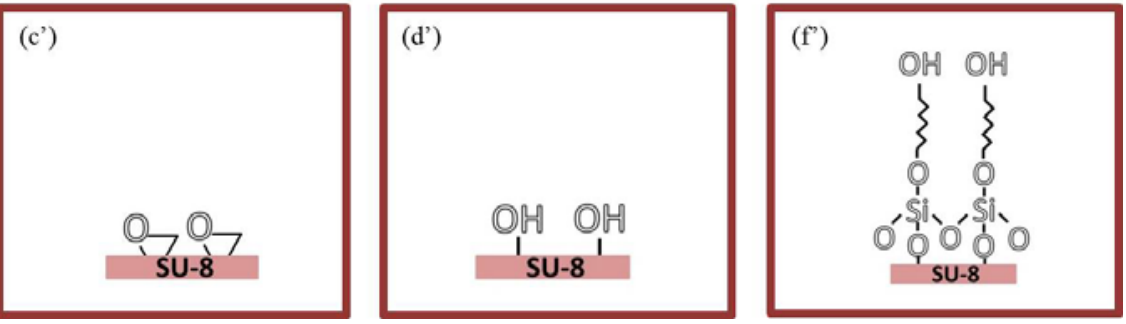

Figure S1. Fabrication(a-c) and modification(d-f) procedure of the PEG-modified TSFs: (c') unmodified SU-8 surface, (d') acid-treated SU-8 surface, and (f') PEG-modified SU-8 surface.

To determine the effect of acid treatment on the TSFs, the filter dimension was measured before and after acid treatment using microscope and MetaMorph ${ }^{\circledR}$ S/W (Molecular Devices, USA). The results are presented in Table S1. The rate of change was negligible compared to fabrication error.

Table S1: The rate of change of tapered-slit SU-8 TSFs during the acid-treating process.

\begin{tabular}{|c|r|r|r|}
\hline $\begin{array}{c}\text { Filter } \\
\text { dimensions }\end{array}$ & \multicolumn{1}{|c|}{$\begin{array}{c}\text { Measured before } \\
\text { acid-treatment } \\
\text { [um] }\end{array}$} & $\begin{array}{l}\text { Measured after } \\
\text { acid-treatment } \\
\text { [um] }\end{array}$ & $\begin{array}{c}\text { Rate of change } \\
\text { [\%] }\end{array}$ \\
\hline$L 1$ & 31.01 & 33.77 & 8.17 \\
\hline$W 1$ & 7.39 & 7.60 & 2.76 \\
\hline$L 2$ & 29.57 & 30.88 & 4.24 \\
\hline$W 2$ & 6.24 & 6.68 & 6.59 \\
\hline
\end{tabular}




\section{S2. XPS Analysis}

The chemical composition of PEG-modified TSFs was characterized by XPS (Thermo VG Scientific, U.S.A.). The Oxygen-to-Carbon (O/C) Ratio of the unmodified SU-8 TSFs, acid-treated SU-8 TSFs, and PEG-modified SU-8 TSFs is described below:

Table S2. X-ray photoelectron spectroscopy (XPS) survey spectra:

(a) unmodified SU-8 TSFs, (b) acid-treated SU-8 TSFs, and (c) PEG-modified SU-8 TSFs.

\begin{tabular}{|c|c|c|c|}
\hline \multirow{2}{*}{ Samples } & \multicolumn{2}{|c|}{$\begin{array}{c}\text { Compensated } \\
\text { Atomic Percentage (\%) }\end{array}$} & \multirow{2}{*}{$\begin{array}{c}\text { Oxygen-to-Carbon } \\
\text { (O/C) Ratio }\end{array}$} \\
\cline { 2 - 3 } & $\mathrm{C} 1 \mathrm{~S}$ & $\mathrm{O} 1 \mathrm{~S}$ & \\
\hline Unmodified SU8 TSFs & 76.96 & 16.83 & 0.22 \\
\hline Acid-treated SU-8 TSFs & 72.44 & 23.78 & 0.33 \\
\hline PEG-modified SU-8 TSFs & 68.73 & 25.38 & 0.37 \\
\hline
\end{tabular}

The XPS high-resolution scans were used to quantify the presence of the PEG on the filter. Fig. S2 shows the growing intensity of the C-O peak (around $286.5 \mathrm{eV}$ ). The Area (P)/CPS.eV were 50616.1, 45820.56, and 42245.62 on the unmodified SU-8 TSFs, acid-treated SU-8 TSFs, and PEG-modified SU-8 TSFs, respectively (Fig.S2-a). On the other hand, The Area (P)/CPS.eV around the $532.0 \mathrm{eV}$ (O-C peak) were 30087.07, 40828.8, and 42425.85 (Fig.S2-b).

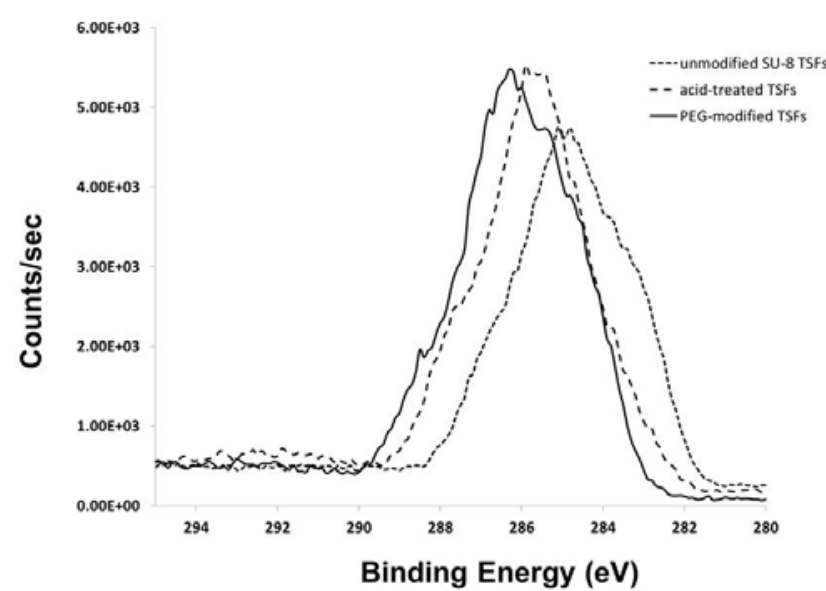

(a)

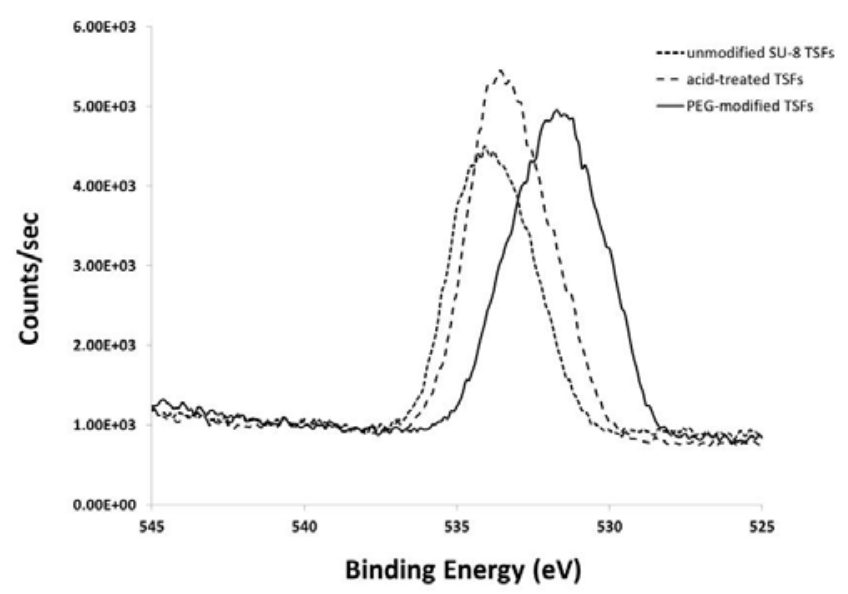

(b)

Figure S2. The XPS spectrum of the unmodified TSFs, acid-treated TSFs, and PEG-modified TSFs: (a) high-resolution spectrum of C1s scans; (b) high-resolution spectrum of O1s scans. 


\section{S3. Purity Test}

In order to verify the performance of the PEG-modified TSFs, purity test was also performed. The purity is defined as follow:

$$
\text { Purity }(\%)=\frac{(\text { The number of the cancer cells })}{(\text { The total number of the released cells })} \times 100(\%)
$$

As it is seen Fig. S3, the results of the unmodified TSFs and the PEG-modified TSFs were $10.52 \pm 4.70 \%$ and $13.08 \pm 5.66 \%$, respectively. In other words, about 10 out of every 100 cells in the released samples were confirmed as cancer cells. There was a slight difference between two types of filters within a margin of error. It means PEG modification did not affect its performance.

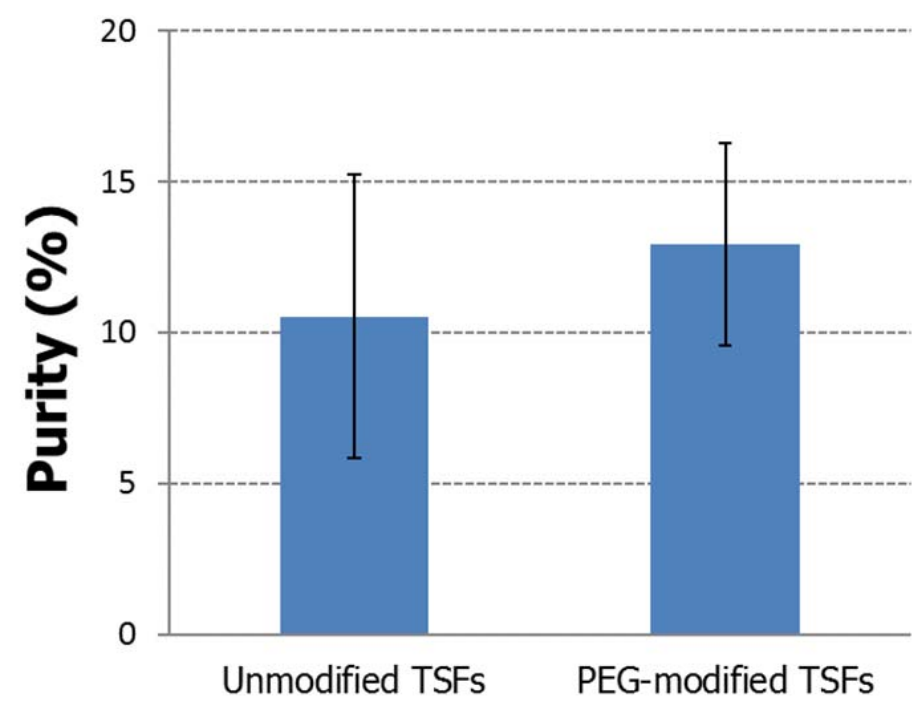

Figure S3. The results of the purity test with the released samples from the unmodified TSFs and the PEG-modified TSFs. The tests were also repeated three separate times $(n=3)$.

It is quite low compared to immunoaffinity-based isolation (e.g. antigen-antibody reaction), but it is comparable (or even higher value) compared to the previous size-based methods. 


\section{S4. Cell Capturing Appearance}

Fluorescent images were obtained after capturing the FITC-dyed cancer cells. As we described earlier, there were significant differences in appearance between unmodified TSFs and PEG-modified TSFs. Taken as a whole, these differences of each cell were not distinguishable; but, when it magnified enough by a microscope, the shape and appearance of the captured cells were differ from each other. On the unmodified TSFs, most average-sized cells showed a tendency of entering into the slit completely; so, the fluorescent image was exaggerated as if the entire slit was filled with a fluorescent substance, like as shown in Fig. S4(a). As matter of fact, the cell occupied top half of the slit. On the other hand, most cells were placed on the edge of the slits. Accordingly, the images of the PEG-modified TSFs were taken different z-position from the unmodified TSFs (That is the reason why the bright field images in Fig. S4-(b) and (d) looks different). A possible explanation for this might be that the captured cells were pushed out from the slits spontaneously: at the very first moment, the cells were presumed to be captured with similar appearance to the cells captured by the unmodified TSFs. However, they might be not retained for a long-term without external force because the cells were not completely osculated with the surfaces due to the osmotic and elastic forces of the PEG-layers. In other words, the most of them were already released from the slits when the reverse-flow applied to the PEG-modified TSFs for a release state. Although the further studies will be needed to better understand the molecular mechanism behind these finding, we have speculated that the retrieval of the captured cells from the PEG-modified TSFs was comparative more straightforward.

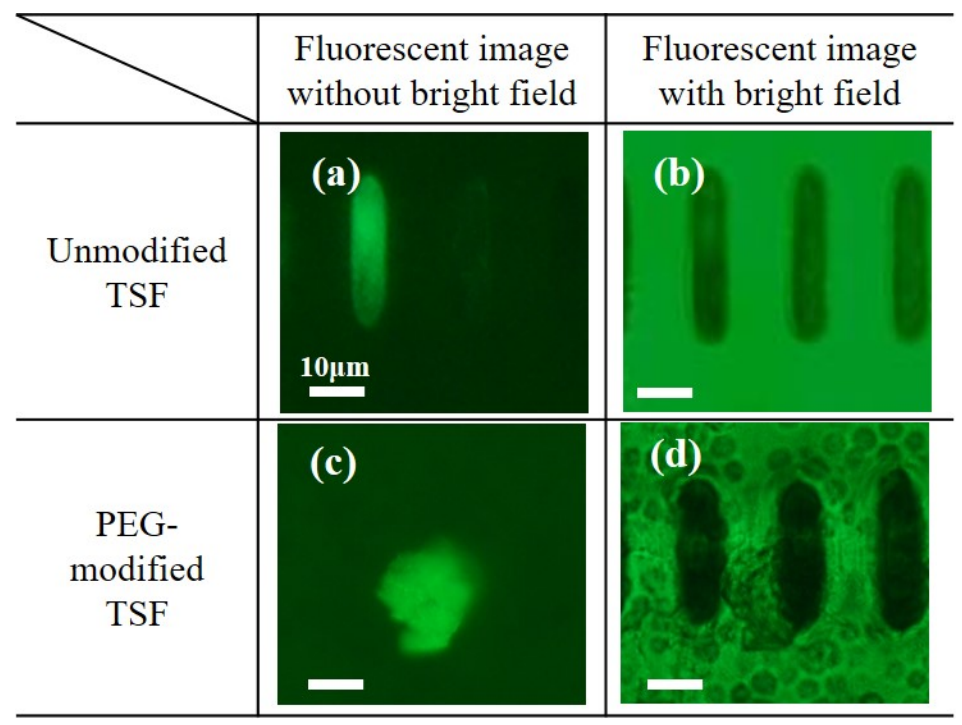

Figure S4. Fluorescence images of the captured cells in the spiked test: $(a, b)$ the fluorescence images on the unmodified TSFs with and without bright field; (c, d) The fluorescence images on the PEGmodified TSFs with and without bright field. (Scale bar=10 $\mu \mathrm{m}$ ) 


\section{S5. Surface Condition Analysis}

The morphology of the surface of the TSFs was examined by Field Emission Scanning Electron Microscopy (FE-SEM). As we noted in the manuscript, there were significant difference in the condition after experiment between the unmodified TSFs and the PEG-modified TSFs. The surface of the unmodified filters was seriously damaged by blood samples; but, the surface of the PEG-modified filters was preserved without damaging or blocking. In the aspect of non-specific blocking, an abundant of cells, including RBCs, WBCs, and cancer cells, were adsorbed on the unmodified filters. However, very little amount of adsorbed cells were found on the PEG-modified filters. This finding has important implications for developing blood compatible medical devices. Further work is required to establish this result.

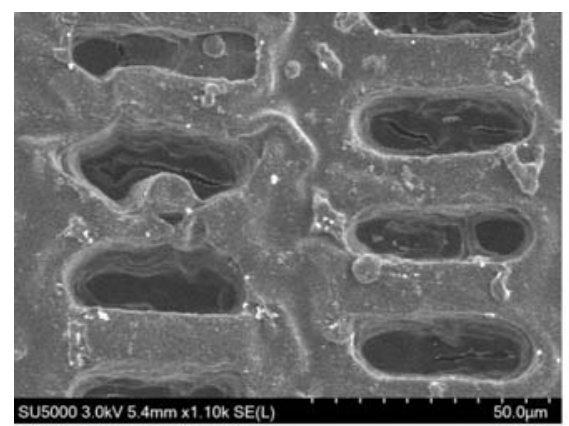

(a)

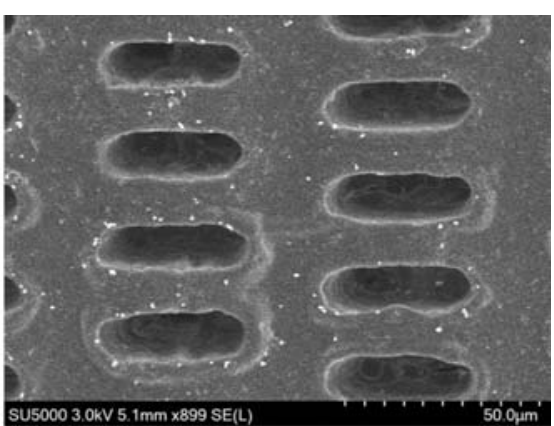

(b)

Fig. S5. The SEM image of the TSFs after a release state:

(a) the unmodified TSFs; (b) the PEG-modified TSFs

There were also some remarkable regions on PEG-modified TSFs (Fig. S6). The cell in Fig.S6-(a) was presumed to be cancer cell which is trapped in the slit. It seems possible that these results are due to clogging problem of the filter-type device. On the other hand, the cell in in Fig.S6-(b) was adsorbed onto the top side of the filter. Since it was flattened for certain reasons, the estimation of the original size of the cell was difficult. It might be adsorbed owing to non-specific adsorption or just remained accidentally on the filters. More valid case of non-specific binding of the cancer cells was presented in Fig.S6-(c): first, it was placed between the slits; second, the shape of the cell was preserved as a globular form; third, the size of the cell was estimated to be 31.4 um. It was definitely even bigger than the biggest blood cells.

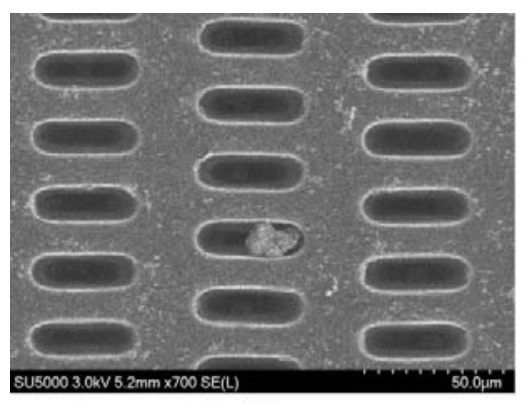

(a)

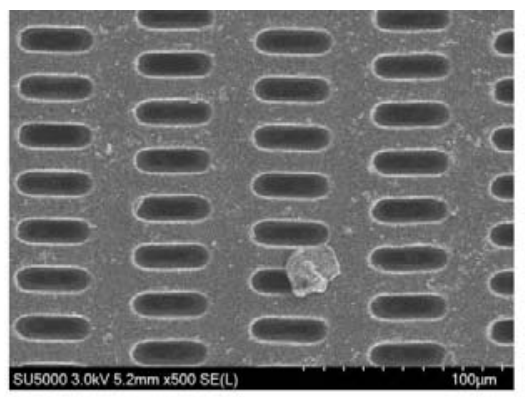

(b)

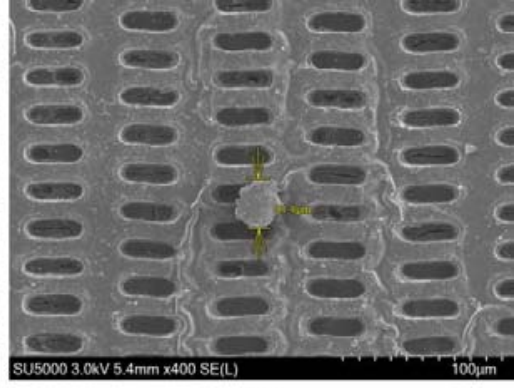

(c)

Figure S6. The cancer cells found on the PEG-modified filters after a release state: (a) the trapped cell inside the slits (magnified by a factor of 700); (b) the absorbed cell onto the slits (magnified by a factor of 500); (c) the absorbed cell between the slits (magnified by a factor of 400). 


\section{S6. RT-PCR Analysis}

Two colorectal cancer patients' samples (CP4, CP5) and one lung cancer patient's sample (LP4) were analyzed by RT-PCR Analysis. The results were explained in the last section of Results and Discussion. EpCAM, CK19, and CD133 were selected for colorectal cancer patients' case. whereas EpCAM, TTF-1, and p63 were selected for lung cancer patients' case.
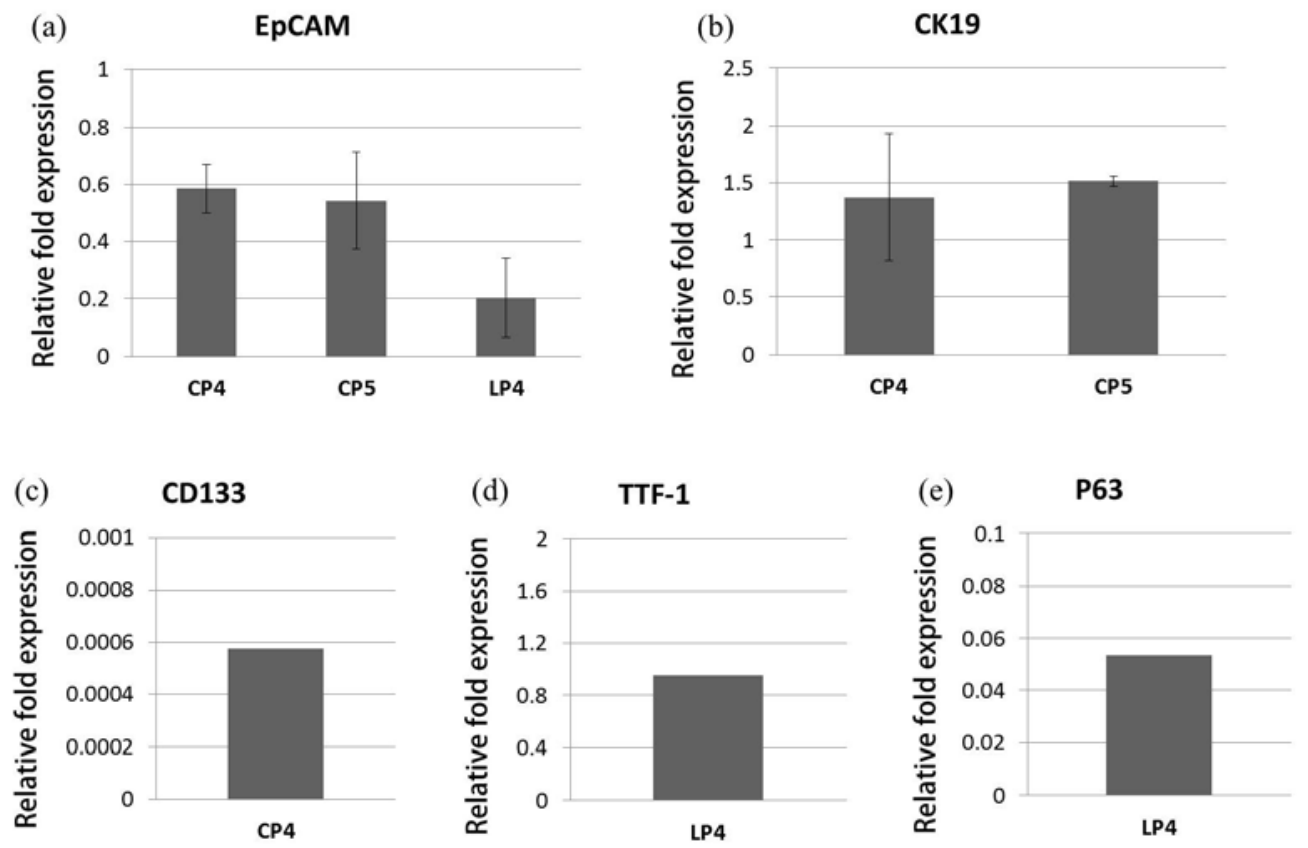

Figure S7. The analysis of marker expression level of the released cells by using RT-PCR:

(a) EpCAM expression; (b) CK19 expression; (c) CD133 expression;

(d) TTF-1 expression; (e) p63 expression. 